\title{
A Generalization on Weighted Means and Convex Functions with respect to the Non-Newtonian Calculus
}

\author{
Ŭgur Kadak $^{1}$ and Yusuf Gürefe ${ }^{2}$ \\ ${ }^{1}$ Department of Mathematics, Bozok University, 66100 Yozgat, Turkey \\ ${ }^{2}$ Department of Econometrics, Uşak University, 64300 Uşak, Turkey \\ Correspondence should be addressed to Uğur Kadak; ugurkadak@gmail.com
}

Received 22 July 2016; Accepted 19 September 2016

Academic Editor: Julien Salomon

Copyright (c) 2016 U. Kadak and Y. Gürefe. This is an open access article distributed under the Creative Commons Attribution License, which permits unrestricted use, distribution, and reproduction in any medium, provided the original work is properly cited.

\begin{abstract}
This paper is devoted to investigating some characteristic features of weighted means and convex functions in terms of the nonNewtonian calculus which is a self-contained system independent of any other system of calculus. It is shown that there are infinitely many such useful types of weighted means and convex functions depending on the choice of generating functions. Moreover, some relations between classical weighted mean and its non-Newtonian version are compared and discussed in a table. Also, some geometric interpretations of convex functions are presented with respect to the non-Newtonian slope. Finally, using multiplicative continuous convex functions we give an application.
\end{abstract}

\section{Introduction}

It is well known that the theory of convex functions and weighted means plays a very important role in mathematics and other fields. There is wide literature covering this topic (see, e.g., [1-8]). Nowadays the study of convex functions has evolved into a larger theory about functions which are adapted to other geometries of the domain and/or obey other laws of comparison of means. Also the study of convex functions begins in the context of real-valued functions of a real variable. More important, they will serve as a model for deep generalizations into the setting of several variables.

As an alternative to the classical calculus, Grossman and Katz [9-11] introduced the non-Newtonian calculus consisting of the branches of geometric, quadratic and harmonic calculus, and so forth. All these calculi can be described simultaneously within the framework of a general theory. They decided to use the adjective non-Newtonian to indicate any calculi other than the classical calculus. Every property in classical calculus has an analogue in non-Newtonian calculus which is a methodology that allows one to have a different look at problems which can be investigated via calculus. In some cases, for example, for wage-rate (in dollars, euro, etc.) related problems, the use of bigeometric calculus which is a kind of non-Newtonian calculus is advocated instead of a traditional Newtonian one.

Many authors have extensively developed the notion of multiplicative calculus; see [12-14] for details. Also some authors have also worked on the classical sequence spaces and related topics by using non-Newtonian calculus [15-17]. Furthermore, Kadak et al. [18, 19] characterized the classes of matrix transformations between certain sequence spaces over the non-Newtonian complex field and generalized RungeKutta method with respect to the non-Newtonian calculus. For more details, see [20-22].

The main focus of this work is to extend weighted means and convex functions based on various generator functions, that is, exp and $q_{p}\left(p \in \mathbb{R}^{+}\right)$generators.

The rest of this paper is organized as follows: in Section 2, we give some required definitions and consequences related with the $\alpha$-arithmetic and $q_{p}$-arithmetic. Based on two arbitrarily selected generators $\alpha$ and $\beta$, we give some basic definitions with respect to the $*$-arithmetic. We also report the most relevant and recent literature in this section. 
In Section 3, first the definitions of non-Newtonian means are given which will be used for non-Newtonian convexity. In this section, the forms of weighted means are presented and an illustrative table is given. In Section 4, the generalized nonNewtonian convex function is defined on the interval $I_{\alpha}$ and some types of convex function are obtained by using different generators. In the final section of the paper, we assert the notion of multiplicative Lipschitz condition on the closed interval $[x, y] \subset(0, \infty)$.

\section{Preliminary, Background, and Notation}

Arithmetic is any system that satisfies the whole of the ordered field axioms whose domain is a subset of $\mathbb{R}$. There are infinitely many types of arithmetic, all of which are isomorphic, that is, structurally equivalent.

A generator $\alpha$ is a one-to-one function whose domain is $\mathbb{R}$ and whose range is a subset $\mathbb{R}_{\alpha}$ of $\mathbb{R}$ where $\mathbb{R}_{\alpha}=\{\alpha(x)$ : $x \in \mathbb{R}\}$. Each generator generates exactly one arithmetic, and conversely each arithmetic is generated by exactly one generator. If $I(x)=x$, for all $x \in \mathbb{R}$, the identity function's inverse is itself. In the special cases $\alpha=I$ and $\alpha=\exp , \alpha$ generates the classical and geometric arithmetic, respectively. By $\alpha$-arithmetic, we mean the arithmetic whose domain is $\mathbb{R}$ and whose operations are defined as follows: for $x, y \in \mathbb{R}_{\alpha}$ and any generator $\alpha$,

$$
\begin{array}{r}
\alpha \text {-addition } x \dot{+} y=\alpha\left\{\alpha^{-1}(x)+\alpha^{-1}(y)\right\}, \\
\alpha \text {-subtraction } x \dot{-} y=\alpha\left\{\alpha^{-1}(x)-\alpha^{-1}(y)\right\}, \\
\alpha \text {-multiplication } x \dot{\times} y=\alpha\left\{\alpha^{-1}(x) \times \alpha^{-1}(y)\right\}, \\
\alpha \text {-division } x \dot{y} y=\alpha\left\{\alpha^{-1}(x) \div \alpha^{-1}(y)\right\}, \\
\alpha \text {-order } x \dot{<} y \Longleftrightarrow \alpha^{-1}(x)<\alpha^{-1}(y) .
\end{array}
$$

As a generator, we choose exp function acting from $\mathbb{R}$ into the set $\mathbb{R}_{\exp }=(0, \infty)$ as follows:

$$
\begin{aligned}
\alpha: \mathbb{R} & \longrightarrow \mathbb{R}_{\exp } \\
x & \longmapsto y=\alpha(x)=e^{x} .
\end{aligned}
$$

It is obvious that $\alpha$-arithmetic reduces to the geometric arithmetic as follows:

$$
\begin{aligned}
& \text { geometric addition } x \dot{+} y=e^{\{\ln x+\ln y\}}=x \cdot y \text {, } \\
& \text { geometric subtraction } x \dot{-} y=e^{\{\ln x-\ln y\}}=x \div y \text {, } \\
& \text { geometric multiplication } x \dot{\times} y=e^{\{\ln x \ln y\}}=x^{\ln y} \\
& \quad=y^{\ln x}
\end{aligned}
$$

geometric division $x \dot{y} y=e^{\{\ln x / \ln y\}}=x^{1 / \ln y}$,

geometric order $x \dot{<} y \Longleftrightarrow \ln (x)<\ln (y)$.

Following Grossman and Katz [10] we give the infinitely many $q_{p}$-arithmetics, of which the quadratic and harmonic arithmetic are special cases for $p=2$ and $p=-1$, respectively. The function $q_{p}: \mathbb{R} \rightarrow \mathbb{R}_{q} \subseteq \mathbb{R}$ and its inverse $q_{p}^{-1}$ are defined as follows $(p \in \mathbb{R} \backslash\{0\})$ :

$$
\begin{aligned}
& q_{p}(x)= \begin{cases}x^{1 / p}, & x>0 \\
0, & x=0 \\
-(-x)^{1 / p}, & x<0,\end{cases} \\
& q_{p}^{-1}(x)= \begin{cases}x^{p}, & x>0 \\
0, & x=0 \\
-(-x)^{p}, & x<0 .\end{cases}
\end{aligned}
$$

It is to be noted that $q_{p}$-calculus is reduced to the classical calculus for $p=1$. Additionally it is concluded that the $\alpha$ summation can be given as follows:

$$
\begin{aligned}
\sum_{k=1}^{n} x_{k} & =\alpha\left\{\sum_{k=1}^{n} \alpha^{-1}\left(x_{k}\right)\right\} \\
& =\alpha\left\{\alpha^{-1}\left(x_{1}\right)+\cdots+\alpha^{-1}\left(x_{n}\right)\right\} \quad \forall x_{k} \in \mathbb{R}^{+} .
\end{aligned}
$$

Definition 1 (see [15]). Let $X=\left(X, d_{\alpha}\right)$ be an $\alpha$-metric space. Then the basic notions can be defined as follows:

(a) A sequence $x=\left(x_{k}\right)$ is a function from the set $\mathbb{N}$ into the set $\mathbb{R}_{\alpha}$. The $\alpha$-real number $x_{k}$ denotes the value of the function at $k \in \mathbb{N}$ and is called the $k$ th term of the sequence.

(b) A sequence $\left(x_{n}\right)$ in $X=\left(X, d_{\alpha}\right)$ is said to be $\alpha$ convergent if, for every given $\varepsilon \dot{>} \dot{0}\left(\varepsilon \in \mathbb{R}_{\alpha}\right)$, there exist an $n_{0}=n_{0}(\varepsilon) \in \mathbb{N}$ and $x \in X$ such that $d_{\alpha}\left(x_{n}, x\right)=\left|x_{n} \dot{-} x\right|_{\alpha} \dot{<} \varepsilon$ for all $n>n_{0}$ and is denoted by ${ }^{\alpha} \lim _{n \rightarrow \infty} x_{n}=x$ or $x_{n} \stackrel{\alpha}{\rightarrow} x$, as $n \rightarrow \infty$.

(c) A sequence $\left(x_{n}\right)$ in $X=\left(X, d_{\alpha}\right)$ is said to be $\alpha$-Cauchy if for every $\varepsilon \dot{\mathcal{D}} \dot{0}$ there is an $n_{0}=n_{0}(\varepsilon) \in \mathbb{N}$ such that $d_{\alpha}\left(x_{n}, x_{m}\right) \dot{<} \varepsilon$ for all $m, n>n_{0}$.

Throughout this paper, we define the $p$ th $\alpha$-exponent $x^{p_{\alpha}}$ and $q$ th $\alpha$-root $x^{(1 / q)_{\alpha}}$ of $x \in \mathbb{R}^{+}$by

$$
\begin{aligned}
x^{2 \alpha} & =x \dot{\times} x=\alpha\left\{\alpha^{-1}(x) \times \alpha^{-1}(x)\right\} \\
& =\alpha\left\{\left[\alpha^{-1}(x)\right]^{2}\right\}, \\
x^{3 \alpha} & =x^{2} \dot{\times} x \\
& =\alpha\left\{\alpha^{-1}\left\{\alpha\left[\alpha^{-1}(x) \times \alpha^{-1}(x)\right]\right\} \times \alpha^{-1}(x)\right\} \\
& =\alpha\left\{\left[\alpha^{-1}(x)\right]^{3}\right\},
\end{aligned}
$$

$$
x^{p_{\alpha}}=x^{(p-1)_{\alpha}} \dot{\times} x=\alpha\left\{\left[\alpha^{-1}(x)\right]^{p}\right\},
$$


and $\sqrt[\alpha]{x}=x^{(1 / 2)_{\alpha}}=y$ provided there exists an $y \in \mathbb{R}_{\alpha}$ such that $y^{2}=x$.

2.1. *-Arithmetic. Suppose that $\alpha$ and $\beta$ are two arbitrarily selected generators and ("star-") also is the ordered pair of arithmetics ( $\beta$-arithmetic and $\alpha$-arithmetic). The sets $\left(\mathbb{R}_{\beta}, \ddot{+}, \ddot{-}, \ddot{x}, \ddot{l}, \ddot{<}\right)$ and $\left(\mathbb{R}_{\alpha}, \dot{+}, \dot{-}, \dot{x}, \dot{l}, \dot{<}\right)$ are complete ordered fields and beta(alpha)-generator generates beta(alpha)arithmetic, respectively. Definitions given for $\beta$-arithmetic are also valid for $\alpha$-arithmetic. Also $\alpha$-arithmetic is used for arguments and $\beta$-arithmetic is used for values; in particular, changes in arguments and values are measured by $\alpha$ differences and $\beta$-differences, respectively.

Let $x \in\left(\mathbb{R}_{\alpha}, \dot{+}, \dot{-}, \dot{x}, \dot{l}, \dot{<}\right)$ and $y \in\left(\mathbb{R}_{\beta}, \ddot{+}, \ddot{-}, \ddot{x}, \ddot{l}, \ddot{<}\right)$ be arbitrarily chosen elements from corresponding arithmetic. Then the ordered pair $(x, y)$ is called a $*$-point and the set of all $*$-points is called the set of $*$-complex numbers and is denoted by $\mathbb{C}^{*}$; that is,

$$
\mathbb{C}^{*}:=\left\{z^{*}=(x, y) \mid x \in \mathbb{R}_{\alpha}, y \in \mathbb{R}_{\beta}\right\} .
$$

Definition 2 (see [17]). (a) The $*$-limit of a function $f$ at an element $a$ in $\mathbb{R}_{\alpha}$ is, if it exists, the unique number $b$ in $\mathbb{R}_{\beta}$ such that

$$
\begin{aligned}
& { }^{*} \lim _{x \rightarrow a} f(x)=b \Longleftrightarrow \\
& \forall \varepsilon \ddot{>0}, \exists \delta \dot{>0} \ni|f(x) \ddot{-} b|_{\beta} \ddot{<} \varepsilon \\
& \qquad x, \varepsilon \in \mathbb{R}_{\alpha},|x \dot{-} a|_{\alpha}<\delta,
\end{aligned}
$$

for $\delta \in \mathbb{R}_{\beta}$, and is written as ${ }^{*} \lim _{x \rightarrow a} f(x)=b$.

A function $f$ is $*$-continuous at a point $a$ in $\mathbb{R}_{\alpha}$ if and only if $a$ is an argument of $f$ and ${ }^{*} \lim _{x \rightarrow a} f(x)=f(a)$. When $\alpha$ and $\beta$ are the identity function $I$, the concepts of $*$-limit and $*$-continuity are reduced to those of classical limit and classical continuity.

(b) The isomorphism from $\alpha$-arithmetic to $\beta$-arithmetic is the unique function $\iota$ (iota) which has the following three properties:

(i) $\iota$ is one to one.

(ii) $\iota$ is from $\mathbb{R}_{\alpha}$ to $\mathbb{R}_{\beta}$.

(iii) For any numbers $u, v \in \mathbb{R}_{\alpha}$,

$$
\begin{aligned}
& \iota(u+v)=\iota(u) \ddot{+} \iota(v) ; \\
& \iota(u \dot{-} v)=\iota(u) \ddot{-} \iota(v) ; \\
& \iota(u \dot{\times} v)=\iota(u) \ddot{\times} \iota(v) ; \\
& \iota(u \dot{\jmath} v)=\iota(u) \ddot{l} \iota(v) .
\end{aligned}
$$

It turns out that $\iota(x)=\beta\left\{\alpha^{-1}(x)\right\}$ for every $x$ in $\mathbb{R}_{\alpha}$ and that $\iota(\dot{n})=\ddot{n}$ for every $\alpha$-integer $\dot{n}$. Since, for example, $u \dot{+} v=\iota^{-1}\{\iota(u) \ddot{+} \iota(v)\}$, it should be clear that any statement in $\alpha$-arithmetic can readily be transformed into a statement in $\beta$-arithmetic.

Definition 3 (see [10]). The following statements are valid:

(i) The $*$-points $P_{1}, P_{2}$, and $P_{3}$ are $*$-collinear provided that at least one of the following holds:

$$
\begin{aligned}
& d^{*}\left(P_{2}, P_{1}\right) \ddot{+} d^{*}\left(P_{1}, P_{3}\right)=d^{*}\left(P_{2}, P_{3}\right), \\
& d^{*}\left(P_{1}, P_{2}\right) \ddot{+} d^{*}\left(P_{2}, P_{3}\right)=d^{*}\left(P_{1}, P_{3}\right), \\
& d^{*}\left(P_{1}, P_{3}\right) \ddot{+} d^{*}\left(P_{3}, P_{2}\right)=d^{*}\left(P_{1}, P_{2}\right) .
\end{aligned}
$$

(ii) A *-line is a set $L$ of at least two distinct points such that, for any distinct points $P_{1}$ and $P_{2}$ in $L$, a point $P_{3}$ is in $L$ if and only if $P_{1}, P_{2}$, and $P_{3}$ are $*$-collinear. When $\alpha=\beta=I$, the $*$-lines are the straight lines in twodimensional Euclidean space.

(iii) The $*$-slope of a $*$-line through the points $\left(a_{1}, b_{1}\right)$ and $\left(a_{2}, b_{2}\right)$ is given by

$$
\begin{aligned}
m^{*} & =\left(b_{2} \ddot{-} b_{1}\right) \ddot{l} \iota\left(a_{2} \dot{-} a_{1}\right) \\
& =\beta\left\{\frac{\beta^{-1}\left(b_{2}\right)-\beta^{-1}\left(b_{1}\right)}{\alpha^{-1}\left(a_{2}\right)-\alpha^{-1}\left(a_{1}\right)}\right\}, \quad\left(a_{1} \neq a_{2}\right),
\end{aligned}
$$

for $a_{1}, a_{2} \in \mathbb{R}_{\alpha}$ and $b_{1}, b_{2} \in \mathbb{R}_{\beta}$.

If the following $*$-limit in (12) exists, we denote it by $f^{*}(t)$, call it the $*$-derivative of $f$ at $t$, and say that $f$ is $*$ differentiable at $t$ (see [19]):

$$
\begin{aligned}
& { }^{*} \lim _{x \rightarrow t}(f(x) \ddot{-} f(t)) \ddot{/} \iota(x-t) \\
& \quad=\lim _{x \rightarrow t} \beta\left\{\frac{\beta^{-1}\{f(x)\}-\beta^{-1}\{f(t)\}}{\alpha^{-1}(x)-\alpha^{-1}(t)}\right\} \\
& \quad=\lim _{x \rightarrow t} \beta\left\{\frac{\beta^{-1}\{f(x)\}-\beta^{-1}\{f(t)\}}{x-t}\right. \\
& \left.\cdot \frac{x-t}{\alpha^{-1}(x)-\alpha^{-1}(t)}\right\}=\beta\left\{\frac{\left(\beta^{-1} \circ f\right)^{\prime}(t)}{\left(\alpha^{-1}\right)^{\prime}(t)}\right\} .
\end{aligned}
$$

\section{Non-Newtonian (Weighted) Means}

Definition 4 ( $\alpha$-arithmetic mean). Consider that $n$ positive real numbers $x_{1}, x_{2}, \ldots, x_{n}$ are given. The $\alpha$-mean (average), 
denoted by $A_{\alpha}$, is the $\alpha$-sum of $x_{n}$ 's $\alpha$-divided by $\dot{n}$ for all $n \in \mathbb{N}$. That is,

$$
\begin{aligned}
A_{\alpha} & =\sum_{\alpha=1}^{n} x_{k} \dot{j} \dot{n}=\sum_{\alpha=1}^{n} \alpha\left\{\frac{\alpha^{-1}\left(x_{k}\right)}{n}\right\} \\
& =\alpha\left\{\frac{\alpha^{-1}\left(x_{1}\right)+\alpha^{-1}\left(x_{2}\right)+\cdots+\alpha^{-1}\left(x_{n}\right)}{n}\right\} .
\end{aligned}
$$

For $\alpha=\exp$, we obtain that

$$
A_{\exp }=\left(\prod_{k=1}^{n} x_{k}\right)^{1 / n}=\left(x_{1} \cdot x_{2} \cdots x_{n}\right)^{1 / n}
$$

Similarly, for $\alpha=q_{p}$, we get

$$
A_{p}=\left(\frac{x_{1}^{p}+x_{2}^{p}+\cdots+x_{n}^{p}}{n}\right)^{1 / p}, \quad p \in \mathbb{R} \backslash\{0\} .
$$

$A_{\text {exp }}$ and $A_{p}$ are called multiplicative arithmetic mean and $p$-arithmetic mean (as usually known $p$-mean), respectively. One can conclude that $A_{p}$ reduces to arithmetic mean and harmonic mean in the ordinary sense for $p=1$ and $p=-1$, respectively.

Remark 5. It is clear that Definition 4 can be written by using various generators. In particular if we take $\beta$-arithmetic instead of $\alpha$-arithmetic then the mean can be defined by

$$
A_{\beta}=\sum_{\beta=1}^{n} x_{k} \ddot{\gamma} \ddot{n}
$$

Definition 6 ( $\alpha$-geometric mean). Let $x_{1}, x_{2}, \ldots, x_{n} \in \mathbb{R}^{+}$. The $\alpha$-geometric mean, namely, $G_{\alpha}$, is $n$th $\alpha$-root of the $\alpha$ product of $\left(x_{n}\right)$ 's:

$$
\begin{aligned}
G_{\alpha} & =\left({ }_{\alpha} \prod_{k=1}^{n} x_{k}\right)^{(1 / n)_{\alpha}}=\alpha\left\{\left(\prod_{k=1}^{n} \alpha^{-1}\left(x_{k}\right)\right)^{1 / n}\right\} \\
& =\alpha\left\{\left(\alpha^{-1}\left(x_{1}\right), \alpha^{-1}\left(x_{2}\right), \ldots, \alpha^{-1}\left(x_{n}\right)\right)^{1 / n}\right\} .
\end{aligned}
$$

We conclude similarly, by taking the generators $\alpha=\exp$ or $\alpha=q_{p}$, that the $\alpha$-geometric mean can be interpreted as follows:

$$
\begin{array}{r}
G_{\text {exp }}=\exp \left\{\left(\ln x_{1}, \ln x_{2}, \ldots, \ln x_{n}\right)^{1 / n}\right\}, \quad\left(x_{n}>1\right) \\
G_{p}=\left\{\left(x_{1}^{p}, x_{2}^{p}, \ldots, x_{n}^{p}\right)^{1 / n}\right\}^{1 / p}=\left(x_{1}, x_{2}, \ldots, x_{n}\right)^{1 / n}, \\
(p \neq 0) .
\end{array}
$$

Definition 8 (weighted $\alpha$-arithmetic mean). Formally, the
weighted $\alpha$-arithmetic mean of a nonempty set of data
$\left\{x_{1}, x_{2}, \ldots, x_{n}\right\}$ with nonnegative weights $\left\{w_{1}, w_{2}, \ldots, w_{n}\right\}$ is
the quantity

Definition 8 (weighted $\alpha$-arithmetic mean). Formally, the
weighted $\alpha$-arithmetic mean of a nonempty set of data
$\left\{x_{1}, x_{2}, \ldots, x_{n}\right\}$ with nonnegative weights $\left\{w_{1}, w_{2}, \ldots, w_{n}\right\}$ is
the quantity

Definition 8 (weighted $\alpha$-arithmetic mean). Formally, the
weighted $\alpha$-arithmetic mean of a nonempty set of data
$\left\{x_{1}, x_{2}, \ldots, x_{n}\right\}$ with nonnegative weights $\left\{w_{1}, w_{2}, \ldots, w_{n}\right\}$ is
the quantity

Definition 8 (weighted $\alpha$-arithmetic mean). Formally, the
weighted $\alpha$-arithmetic mean of a nonempty set of data
$\left\{x_{1}, x_{2}, \ldots, x_{n}\right\}$ with nonnegative weights $\left\{w_{1}, w_{2}, \ldots, w_{n}\right\}$ is
the quantity

$G_{\text {exp }}$ and $G_{p}$ are called multiplicative geometric mean and $p$-geometric mean, respectively. It would clearly have $G_{p}=$ $A_{\text {exp }}$ for $p=1$.

Definition 7 ( $\alpha$-harmonic mean). Let $x_{1}, x_{2}, \ldots, x_{n} \in \mathbb{R}^{+}$and $\alpha^{-1}\left(x_{n}\right) \neq 0$ for each $n \in \mathbb{N}$. The $\alpha$-harmonic mean $H_{\alpha}$ is defined by

$$
\begin{aligned}
H_{\alpha} & =\dot{n} / \sum_{\alpha=1}^{n}\left(\mathrm{i} / x_{k}\right) \\
& =\dot{n} \dot{j}\left(\dot{\mathrm{i}} / x_{1} \dot{+} \mathrm{i} / x_{2} \dot{+} \cdots \dot{\mathrm{i}} / x_{n}\right) \\
& =\alpha\left\{\frac{n}{1 / \alpha^{-1}\left(x_{1}\right)+1 / \alpha^{-1}\left(x_{2}\right)+\cdots+1 / \alpha^{-1}\left(x_{n}\right)}\right\} .
\end{aligned}
$$

Similarly, one obtains that

$$
\begin{aligned}
H_{\text {exp }} & \\
= & \exp \left\{\frac{n}{1 / \ln \left(x_{1}\right)+1 / \ln \left(x_{2}\right)+\cdots+1 / \ln \left(x_{n}\right)}\right\}, \\
H_{p} & =\left\{\frac{n}{\left.1 /\left(x_{1}\right)^{p}+1\right),\left(x_{2}\right)^{p}+\cdots+1 /\left(x_{n}\right)^{p}}\right\}^{1 / p} .
\end{aligned}
$$

$H_{\text {exp }}$ and $H_{p}$ are called multiplicative harmonic mean and $p$ harmonic mean, respectively. Obviously the inclusion (20) is reduced to ordinary harmonic mean and ordinary arithmetic mean for $p=1$ and $p=-1$, respectively.

3.1. Non-Newtonian Weighted Means. The weighted mean is similar to an arithmetic mean, where instead of each of the data points contributing equally to the final average, some data points contribute more than others. Moreover the notion of weighted mean plays a role in descriptive statistics and also occurs in a more general form in several other areas of mathematics.

The following definitions can give the relationships between the non-Newtonian weighted means and ordinary weighted means. 


$$
\widetilde{A}_{\alpha}=\sum_{\alpha=1}^{n}\left(x_{i} \dot{\times} \dot{w}_{i}\right) \dot{\alpha} \sum_{i=1}^{n} \dot{w}_{i}=\left(x_{1} \dot{\times} \dot{w}_{1} \dot{+} x_{2} \dot{\times} \dot{w}_{2} \dot{+} \cdots \dot{+} x_{n} \dot{\times} \dot{w}_{n}\right) \dot{j}\left(\dot{w}_{1} \dot{+} \dot{w}_{2} \dot{+} \cdots \dot{+} \dot{w}_{n}\right)=\alpha\left\{\frac{\sum_{i=1}^{n} w_{i} \alpha^{-1}\left(x_{i}\right)}{\sum_{i=1}^{n} w_{i}}\right\} .
$$

The formulas are simplified when the weights are $\alpha$ normalized such that they $\alpha$-sum up to $\sum_{\alpha}^{n} \sum_{i=1}^{n} \dot{w}_{i}=\dot{1}$. For such normalized weights the weighted $\alpha$-arithmetic mean is simply $\widetilde{A}_{\alpha}={ }_{\alpha} \sum_{i=1}^{n} x_{i} \dot{\times} \dot{w}_{i}$. Note that if all the weights are equal, the weighted $\alpha$-arithmetic mean is the same as the $\alpha$ arithmetic mean.

Taking $\alpha=\exp$ and $\alpha=q_{p}$, the weighted $\alpha$-arithmetic mean can be given with the weights $\left\{w_{1}, w_{2}, \ldots, w_{n}\right\}$ as follows:

$$
\begin{aligned}
& \widetilde{A}_{\exp }=\exp \left\{\frac{\sum_{i=1}^{n} \ln \left(x_{i}\right) w_{i}}{\sum_{i=1}^{n} w_{i}}\right\}=\left(\prod_{i=1}^{n} x_{i}^{w_{i}}\right)^{1 / \sum_{i=1}^{n} w_{i}}, \\
& \widetilde{A}_{q_{p}}=\left\{\frac{w_{1}\left(x_{1}\right)^{p}+w_{2}\left(x_{2}\right)^{p}+\cdots+w_{n}\left(x_{n}\right)^{p}}{w_{1}+w_{2}+\cdots+w_{n}}\right\}^{1 / p} .
\end{aligned}
$$

$\widetilde{A}_{\text {exp }}$ and $\widetilde{A}_{p}$ are called multiplicative weighted arithmetic mean and weighted $p$-arithmetic mean, respectively. $\widetilde{A}_{\text {exp }}$ turns out to the ordinary weighted geometric mean. Also, one easily can see that $\widetilde{A}_{p}$ is reduced to ordinary weighted arithmetic mean and weighted harmonic mean for $p=1$ and $p=-1$, respectively.

Definition 9 (weighted $\alpha$-geometric mean). Given a set of positive reals $\left\{x_{1}, x_{2}, \ldots, x_{n}\right\}$ and corresponding weights $\left\{w_{1}, w_{2}, \ldots, w_{n}\right\}$, then the weighted $\alpha$-geometric mean $\widetilde{G}_{\alpha}$ is defined by

$$
\begin{aligned}
\widetilde{G}_{\alpha} & =\left\{\alpha \prod_{i=1}^{n} x_{i}\right\}^{\left(w_{i} / \sum_{i=1}^{n} w_{i}\right)_{\alpha}}=x_{1}^{\left(w_{1} /\left(w_{1}+\cdots+w_{n}\right)\right)_{\alpha}} \\
\dot{\times} & x_{2}^{\left(w_{2} /\left(w_{1}+\cdots+w_{n}\right)\right)_{\alpha}} \dot{\times} \cdots \dot{\times} x_{n}^{\left(w_{n} /\left(w_{1}+\cdots+w_{n}\right)\right)_{\alpha}} \\
& =\alpha\left\{\left[\alpha^{-1}\left(x_{1}\right)\right]^{w_{1} /\left(w_{1}+\cdots+w_{n}\right)},\right. \\
& {\left.\left[\alpha^{-1}\left(x_{2}\right)\right]^{w_{2} /\left(w_{1}+\cdots+w_{n}\right)}, \ldots,\left[\alpha^{-1}\left(x_{n}\right)\right]^{w_{n} /\left(w_{1}+\cdots+w_{n}\right)}\right\} . }
\end{aligned}
$$

Note that if all the weights are equal, the weighted $\alpha$ geometric mean is the same as the $\alpha$-geometric mean. Taking $\alpha=\exp$ and $\alpha=q_{p}$, the weighted $\alpha$-geometric mean can be written for the weights $\left\{w_{1}, w_{2}, \ldots, w_{n}\right\}$ as follows:

$$
\begin{gathered}
\widetilde{G}_{\exp }=\exp \left\{\left[\ln \left(x_{1}\right)\right]^{w_{1} /\left(w_{1}+\cdots+w_{n}\right)},\right. \\
\left.\left[\ln \left(x_{2}\right)\right]^{w_{2} /\left(w_{1}+\cdots+w_{n}\right)}, \ldots,\left[\ln \left(x_{n}\right)\right]^{w_{n} /\left(w_{1}+\cdots+w_{n}\right)}\right\}, \\
\widetilde{G}_{p}=\left\{x_{n}^{p w_{1} /\left(w_{1}+\cdots+w_{n}\right)}, x_{2}^{p w_{2} /\left(w_{1}+\cdots+w_{n}\right)}, \ldots,\right. \\
\left.x_{n}^{p w_{n} /\left(w_{1}+\cdots+w_{n}\right)}\right\}^{1 / p}=\left(\prod_{i=1}^{n} x_{i}^{w_{i}}\right)^{1 / \sum_{i=1}^{n} w_{i}} .
\end{gathered}
$$

$\widetilde{G}_{\text {exp }}$ and $\widetilde{G}_{p}$ are called weighted multiplicative geometric mean and weighted $q$-geometric mean. Also we have $\widetilde{G}_{p}=$ $\widetilde{A}_{\text {exp }}$ for all $x_{n}>1$.

Definition 10 (weighted $\alpha$-harmonic mean). If a set $\left\{w_{1}\right.$, $\left.w_{2}, \ldots, w_{n}\right\}$ of weights is associated with the data set $\left\{x_{1}, x_{2}, \ldots, x_{n}\right\}$ then the weighted $\alpha$-harmonic mean is defined by

$$
\begin{aligned}
& \widetilde{H}_{\alpha}=\sum_{\alpha=1}^{n} \dot{w}_{k} \dot{j} \sum_{k=1}^{n}\left(\dot{w}_{k} \dot{j} x_{k}\right) \\
& =\left(\dot{w}_{1} \dot{+} \cdots \dot{+} \dot{w}_{n}\right) \dot{/}\left(\dot{w}_{1} \dot{j} x_{1} \dot{+} \dot{w}_{2} / x_{2} \dot{+} \cdots \dot{+} \dot{w}_{n} / x_{n}\right) \\
& =\alpha\left\{\frac{w_{1}+w_{2}+\cdots+w_{n}}{w_{1} / \alpha^{-1}\left(x_{1}\right)+w_{2} / \alpha^{-1}\left(x_{2}\right)+\cdots+w_{n} / \alpha^{-1}\left(x_{n}\right)}\right\} .
\end{aligned}
$$

Taking $\alpha=\exp$ and $\alpha=q_{p}$, the weighted $\alpha$-harmonic mean with the weights $\left\{w_{1}, w_{2}, \ldots, w_{n}\right\}$ can be written as follows:

$$
\begin{aligned}
& \widetilde{H}_{\exp } \\
& \quad=\exp \left\{\frac{w_{1}+w_{2}+\cdots+w_{n}}{w_{1} / \ln x_{1}+w_{2} / \ln x_{2}+\cdots+w_{n} / \ln x_{n}}\right\}, \\
& \widetilde{H}_{p} \quad\left(x_{n}>1\right), \\
& \quad=\left\{\frac{w_{1}+w_{2}+\cdots+w_{n}}{w_{1} /\left(x_{1}\right)^{p}+w_{2} /\left(x_{2}\right)^{p}+\cdots+w_{n} /\left(x_{n}\right)^{p}}\right\}^{1 / p} .
\end{aligned}
$$

$\widetilde{H}_{\text {exp }}$ and $\widetilde{H}_{p}$ are called multiplicative weighted harmonic mean and weighted $p$-geometric mean, respectively. It is obvious that $\widetilde{H}_{p}$ is reduced to ordinary weighted harmonic mean and ordinary weighted arithmetic mean for $p=1$ and $p=-1$, respectively. 
TABLE 1: Comparison of the non-Newtonian (weighted) means and ordinary (weighted) means.

\begin{tabular}{|c|c|c|c|c|c|c|c|c|c|c|c|c|}
\hline Weight & Data & $p$ & $A_{p}$ & $G_{p}$ & $H_{p}$ & $\widetilde{A}_{p}$ & $\widetilde{G}_{p}$ & $\widetilde{H}_{p}$ & $G_{\exp }$ & $H_{\exp }$ & $\widetilde{G}_{\exp }$ & $\widetilde{H}_{\exp }$ \\
\hline$w_{1}=2$ & $x_{1}=15$ & 1.0 & 27.50 & 24.74 & 22.64 & 32.82 & 29.88 & 27.27 & 24.02 & 23.36 & 29.05 & 28.26 \\
\hline$w_{2}=5$ & $x_{2}=20$ & 0.1 & 24.99 & 24.74 & 24.50 & 30.16 & 29.88 & 29.59 & 24.02 & 23.36 & 29.05 & 28.26 \\
\hline$w_{3}=7$ & $x_{3}=25$ & 2.0 & 30.61 & 24.74 & 21.14 & 35.77 & 29.88 & 25.21 & 24.02 & 23.36 & 29.05 & 28.26 \\
\hline$w_{4}=9$ & $x_{4}=50$ & 5.0 & 38.22 & 24.74 & 18.73 & 41.69 & 29.88 & 21.55 & 24.02 & 23.36 & 29.05 & 28.26 \\
\hline
\end{tabular}

In Table 1 , the non-Newtonian means are obtained by using different generating functions. For $\alpha=q_{p}$, the $p$-means $A_{p}, G_{p}$ and $H_{p}$ are reduced to ordinary arithmetic mean, geometric mean, and harmonic mean, respectively. In particular some changes are observed for each value of $A_{p}, G_{p}$, and $H_{p}$ means depending on the choice of $p$. As shown in the table, for increasing values of $p$, the $p$-arithmetic mean $A_{p}$ and its weighted form $\widetilde{A}_{p}$ increase; in particular $p$ tends to $\infty$, and these means converge to the value of $\max \left\{x_{n}\right\}$. Conversely, for increasing values of $p$, the $p$-harmonic mean $H_{p}$ and its weighted forms $\widetilde{H}_{p}$ decrease. In particular, these means converge to the value of $\min \left\{x_{n}\right\}$ as $p \rightarrow \infty$. Depending on the choice of $p$, weighted forms $\widetilde{A}_{p}$ and $\widetilde{H}_{p}$, can be increased or decreased without changing any weights. For this reason, this approach brings a new perspective to the concept of classical (weighted) mean. Moreover, when we compare $H_{\text {exp }}$ and ordinary harmonic mean in Table 1 , we also see that ordinary harmonic mean is smaller than $H_{\text {exp }}$. On the contrary $A_{\exp }$ and $G_{\exp }$ are smaller than their classical forms $A_{p}$ and $G_{p}$ for $p=1$. Therefore, we assert that the values of $G_{\text {exp }}, H_{\text {exp }}, \widetilde{G}_{\text {exp }}$, and $\widetilde{H}_{\exp }$ should be evaluated satisfactorily.

Corollary 11. Consider $n$ positive real numbers $x_{1}, x_{2}, \ldots, x_{n}$. Then, the conditions $H_{\alpha}<G_{\alpha}<A_{\alpha}$ and $\widetilde{H}_{\alpha}<\widetilde{G}_{\alpha}<\widetilde{A}_{\alpha}$ hold when $\alpha=\exp$ for all $x_{n}>1$ and $\alpha=q_{p}$ for all $p \in \mathbb{R}^{+}$.

\section{Non-Newtonian Convexity}

In this section, the notion of non-Newtonian convex $(*-$ convex) functions will be given by using different generators. Furthermore the relationships between $*$-convexity and non-Newtonian weighted mean will be determined.

Definition 12 (generalized $*$-convex function). Let $I_{\alpha}$ be an interval in $\mathbb{R}_{\alpha}$. Then $f: I_{\alpha} \rightarrow \mathbb{R}_{\beta}$ is said to be $*$-convex if

$$
f\left(\lambda_{1} \dot{x} x \dot{+} \lambda_{2} \dot{x} y\right) \ddot{\leq} \mu_{1} \ddot{\times} f(x) \ddot{+} \mu_{2} \ddot{x} f(y)
$$

holds, where $\lambda_{1} \dot{+} \lambda_{2}=\mathrm{i}$ and $\mu_{1} \ddot{+} \mu_{2}=\ddot{1}$ for all $\lambda_{1}, \lambda_{2} \epsilon$ $[\dot{0}, \dot{1}]$ and $\mu_{1}, \mu_{2} \in[\ddot{0}, \ddot{1}]$. Therefore, by combining this with the generators $\alpha$ and $\beta$, we deduce that

$$
\begin{aligned}
& f\left(\alpha\left\{\alpha^{-1}\left(\lambda_{1}\right) \alpha^{-1}(x)+\alpha^{-1}\left(\lambda_{2}\right) \alpha^{-1}(y)\right\}\right) \\
& \quad \ddot{\leq} \beta\left\{\beta^{-1}\left(\mu_{1}\right) \beta^{-1} f(x)+\beta^{-1}\left(\mu_{2}\right) \beta^{-1} f(y)\right\} .
\end{aligned}
$$

If (28) is strict for all $x \neq y$, then $f$ is said to be strictly $*$ convex. If the inequality in (28) is reversed, then $f$ is said to be $*$-concave. On the other hand the inclusion (28) can be written with respect to the weighted $\alpha$-arithmetic mean in (21) as follows:

$$
f\left(\widetilde{A}_{\alpha}\{x, y\}\right) \ddot{\leq} \widetilde{A}_{\beta}\{f(x), f(y)\} .
$$

Remark 13 . We remark that the definition of $*$-convexity in (27) can be evaluated by non-Newtonian coordinate system involving $*$-lines (see Definition 3). For $\alpha=\beta=I$, the $*$ lines are straight lines in two-dimensional Euclidean space. For this reason, we say that almost all the properties of ordinary Cartesian coordinate system will be valid for nonNewtonian coordinate system under $*$-arithmetic.

Also depending on the choice of generator functions, the definition of $*$-convexity in (27) can be interpreted as follows.

Case 1. (a) If we take $\alpha=\beta=\exp$ and $\lambda_{1}=\mu_{1}, \lambda_{2}=\mu_{2}$ in (28), then

$$
\begin{aligned}
f\left(x^{\ln \lambda_{1}} y^{\ln \lambda_{2}}\right) \leq f(x)^{\ln \lambda_{1}} f(y)^{\ln \lambda_{2}}, & \\
& \left(\lambda_{1}, \lambda_{2} \in[1, e]\right),
\end{aligned}
$$

where $\lambda_{1} \lambda_{2}=e$ holds and $f: I_{\exp } \rightarrow \mathbb{R}_{\exp }=(0, \infty)$ is called bigeometric (usually known as multiplicative) convex function (cf. [2]). Equivalently, $f$ is bigeometric convex if and only if $\log f(x)$ is an ordinary convex function.

(b) For $\alpha=\exp$ and $\beta=I$ we have

$$
\begin{aligned}
& f\left(x^{\ln \lambda_{1}} y^{\ln \lambda_{2}}\right) \leq \mu_{1} f(x)+\mu_{2} f(y), \\
& \left(\lambda_{1}, \lambda_{2} \in[1, e] ; \mu_{1}, \mu_{2} \in[0,1]\right),
\end{aligned}
$$

where $\lambda_{1} \lambda_{2}=e$ and $\mu_{1}+\mu_{2}=1$. In this case the function $f$ : $I_{\exp } \rightarrow \mathbb{R}$ is called geometric convex function. Every geometric convex (usually known as log-convex) function is also convex (cf. [2]).

(c) Taking $\alpha=I$ and $\beta=$ exp, one obtains

$$
\begin{aligned}
& f\left(\lambda_{1} x+\lambda_{2} y\right) \leq f(x)^{\ln \mu_{1}} f(y)^{\ln \mu_{2}}, \\
& \left(\mu_{1}, \mu_{2} \in[1, e] ; \lambda_{1}, \lambda_{2} \in[0,1]\right),
\end{aligned}
$$

where $\mu_{1} \mu_{2}=e$ and $\lambda_{1}+\lambda_{2}=1$, and $f: I \rightarrow \mathbb{R}_{\exp }$ is called anageometric convex function.

Case 2. (a) If $\alpha=\beta=q_{p}$ in (28) then

$$
\begin{aligned}
& f\left(\left(\left(\lambda_{1} x\right)^{p}+\left(\lambda_{2} y\right)^{p}\right)^{1 / p}\right) \\
& \quad \leq\left(\left(\lambda_{1} f(x)\right)^{p}+\left(\lambda_{2} f(y)\right)^{p}\right)^{1 / p} \quad\left(p \in \mathbb{R}^{+}\right),
\end{aligned}
$$


where $\lambda_{1}, \lambda_{2} \in[0,1], \lambda_{1}^{p}+\lambda_{2}^{p}=1$, and $f: I_{q_{p}} \rightarrow \mathbb{R}_{q_{p}}$ is called $Q Q$-convex function.

(b) For $\alpha=q_{p}$ and $\beta=I$, we write that

$$
\begin{aligned}
f\left(\left(\left(\lambda_{1} x\right)^{p}+\left(\lambda_{2} y\right)^{p}\right)^{1 / p}\right) \leq & \mu_{1} f(x)+\mu_{2} f(y), \\
& \left(\lambda_{1}, \lambda_{2}, \mu_{1}, \mu_{2} \in[0,1]\right),
\end{aligned}
$$

where $\lambda_{1}^{p}+\lambda_{2}^{p}=1, \mu_{1}+\mu_{2}=1$, and $f: I_{q_{p}} \rightarrow \mathbb{R}$ is called $Q I$-convex function.

(c) For $\alpha=I$ and $\beta=q_{p}$, we obtain that

$$
\begin{aligned}
f\left(\lambda_{1} x+\lambda_{2} y\right) \leq\left(\left(\mu_{1} f(x)\right)^{p}+\right. & \left.\left(\mu_{2} f(y)\right)^{p}\right)^{1 / p}, \\
& \left(\lambda_{1}, \lambda_{2}, \mu_{1}, \mu_{2} \in[0,1]\right),
\end{aligned}
$$

where $\mu_{1}^{p}+\mu_{2}^{p}=1, \lambda_{1}+\lambda_{2}=1$, and $f: I \rightarrow \mathbb{R}_{q_{p}}$ is called IQconvex function.

The *-convexity of a function $f: I_{\alpha} \rightarrow \mathbb{R}_{\beta}$ means geometrically that the *-points of the graph of $f$ are under the chord joining the endpoints $(a, f(a))$ and $(b, f(b))$ on nonNewtonian coordinate system for every $a, b \in I_{\alpha}$. By taking into account the definition of $*$-slope in Definition 3 we have

$$
\begin{aligned}
& (f(x) \ddot{-} f(a)) \ddot{/} \iota(x \dot{-} a) \\
& \quad \ddot{\leq}(f(b) \ddot{-} f(a)) \ddot{/} \iota(b \dot{-} a)
\end{aligned}
$$

which implies

$$
\begin{gathered}
f(x) \ddot{\leq} f(a) \ddot{+}((f(b) \ddot{-} f(a)) \ddot{/} \iota(b \dot{-} a)) \\
\ddot{\times} \iota(x \dot{-} a)
\end{gathered}
$$

for all $x \in[\dot{a}, \dot{b}]$.

On the other hand (37) means that if $P, Q$, and $R$ are any three *-points on the graph of $f$ with $Q$ between $P$ and $R$, then $Q$ is on or below chord $P R$. In terms of $*$-slope, it is equivalent to

$$
m^{*}(P Q) \ddot{\leq} m^{*}(P R) \ddot{\leq} m^{*}(Q R)
$$

with strict inequalities when $f$ is strictly $*$-convex.

Now to avoid the repetition of the similar statements, we give some necessary theorems and lemmas.

Lemma 14 (Jensen's inequality). A $\beta$-real-valued function $f$ defined on an interval $I_{\alpha}$ is $*$-convex if and only if

$$
f\left(\sum_{\alpha=1}^{n} \lambda_{k} \dot{\times} x_{k}\right) \ddot{\leq} \sum_{\beta=1}^{n} \mu_{k} \ddot{x} f\left(x_{k}\right)
$$

holds, where $\sum_{\alpha=1}^{n} \lambda_{k}=\mathrm{i}$ and $\sum_{k=1}^{n} \mu_{k}=\ddot{1}$ for all $\lambda_{n} \in[\dot{0}, \dot{1}]$ and $\mu_{n} \in[\ddot{0}, \ddot{1}]$.

Proof. The proof is straightforward, hence omitted.
Theorem 15. Let $f: I_{\alpha} \rightarrow \mathbb{R}_{\beta}$ be a*-continuous function. Then $f$ is *-convex if and only if $f$ is midpoint *-convex, that is,

$$
\begin{gathered}
x_{1}, x_{2} \in I_{\alpha} \text { implies } \\
f\left(A_{\alpha}\left\{x_{1}, x_{2}\right\}\right) \ddot{\leq} A_{\beta}\left\{f\left(x_{1}\right), f\left(x_{2}\right)\right\} .
\end{gathered}
$$

Proof. The proof can be easily obtained using the inequality (39) in Lemma 14.

Theorem 16 (cf. [2]). Let $f: I_{\exp } \rightarrow \mathbb{R}_{\exp }$ be a*-differentiable function (see [19]) on a subinterval $I_{\exp } \subseteq(0, \infty)$. Then the following assertions are equivalent:

(i) $f$ is bigeometric convex (concave).

(ii) The function $f^{*}(x)$ is increasing (decreasing).

Corollary 17. A positive $\beta$-real-valued function $f$ defined on an interval $I_{\exp }$ is bigeometric convex if and only if

$$
\begin{aligned}
& f\left(x_{1}^{\ln \lambda_{1}}, x_{2}^{\ln \lambda_{2}}, \ldots, x_{n}^{\ln \lambda_{n}}\right) \\
& \quad \leq f\left(x_{1}\right)^{\ln \lambda_{1}}, f\left(x_{2}\right)^{\ln \lambda_{2}}, \ldots, f\left(x_{n}\right)^{\ln \lambda_{n}}
\end{aligned}
$$

holds, where $\prod_{k=1}^{n} \lambda_{k}=e$ for all $x_{1}, x_{2}, \ldots, x_{n} \in I_{\exp }$ and $\lambda_{1}, \lambda_{2}, \ldots, \lambda_{n} \in[1, e]$. Besides, we have

$$
\begin{aligned}
f & \left(\widetilde{A}_{\exp }\left\{x_{1}, x_{2}, \ldots, x_{n}\right\}\right) \\
& \leq \widetilde{A}_{\exp }\left\{f\left(x_{1}\right), f\left(x_{2}\right), \ldots, f\left(x_{n}\right)\right\} .
\end{aligned}
$$

Corollary 18. A $\beta$-real-valued function $f$ defined on an interval $I_{q_{p}}$ is QQ-convex if and only if

$$
\begin{aligned}
& f\left[\left(\left(\lambda_{1} x_{1}\right)^{p}+\left(\lambda_{2} x_{2}\right)^{p}+\cdots+\left(\lambda_{n} x_{n}\right)^{p}\right)^{1 / p}\right] \\
& \quad \leq\left(\left(\lambda_{1} f\left(x_{1}\right)\right)^{p}+\left(\lambda_{2} f\left(x_{2}\right)\right)^{p}+\cdots\right. \\
& \left.\quad+\left(\lambda_{n} f\left(x_{n}\right)\right)^{p}\right)^{1 / p}
\end{aligned}
$$

holds, where $\sum_{k=1}^{n} \lambda_{k}^{p}=1$ for all $x_{1}, x_{2}, \ldots, x_{n} \in I_{q_{p}}$ and $\lambda_{1}, \lambda_{2}, \ldots, \lambda_{n} \in[0,1]$. Thus, we have

$$
\begin{aligned}
f & \left(\widetilde{A}_{p}\left\{x_{1}, x_{2}, \ldots, x_{n}\right\}\right) \\
& \leq \widetilde{A}_{p}\left\{f\left(x_{1}\right), f\left(x_{2}\right), \ldots, f\left(x_{n}\right)\right\}, \quad\left(p \in \mathbb{R}^{+}\right) .
\end{aligned}
$$

\section{An Application of Multiplicative Continuity}

In this section based on the definition of bigeometric convex function and multiplicative continuity, we get an analogue of ordinary Lipschitz condition on any closed interval.

Let $f$ be a bigeometric (multiplicative) convex function and finite on a closed interval $[x, y] \subset \mathbb{R}^{+}$. It is obvious that $f$ is bounded from above by $M=\max \{f(x), f(y)\}$, since, for any $z=x^{\lambda} y^{1-\lambda}$ in the interval, $f(z) \leq f(x)^{\lambda} f(y)^{1-\lambda}$ for 
$\lambda \in[1, e]$. It is also bounded from below as we see by writing an arbitrary point in the form $t \sqrt{x y}$ for $t \in \mathbb{R}^{+}$. Then

$$
f^{2}(\sqrt{x y}) \leq f(t \sqrt{x y}) f\left(\frac{\sqrt{x y}}{t}\right) .
$$

Using $M$ as the upper bound $f(\sqrt{x y} / t)$ we obtain

$$
f(t \sqrt{x y}) \geq \frac{1}{M} f^{2}(\sqrt{x y})=m .
$$

Thus a bigeometric convex function may not be continuous at the boundary points of its domain. We will prove that, for any closed subinterval $[x, y]$ of the interior of the domain, there is a constant $K>0$ so that, for any two points $a, b \in[x, y] \subset$ $\mathbb{R}^{+}$

$$
\frac{f(a)}{f(b)} \leq\left(\frac{a}{b}\right)^{K}
$$

A function that satisfies (47) for some $K$ and all $a$ and $b$ in an interval is said to satisfy bigeometric Lipschitz condition on the interval.

Theorem 19. Suppose that $f: I \rightarrow \mathbb{R}^{+}$is multiplicative convex. Then, $f$ satisfies the multiplicative Lipschitz condition on any closed interval $[x, y] \subset \mathbb{R}^{+}$contained in the interior $I^{0}$ of $I$; that is, $f$ is continuous on $I^{0}$.

Proof. Take $\varepsilon>1$ so that $[x / \varepsilon, y \varepsilon] \in I$, and let $m$ and $M$ be the lower and upper bounds for $f$ on $[x / \varepsilon, y \varepsilon]$. If $r$ and $s$ are distinct points of $[x, y]$ with $s>r$, set

$$
\begin{aligned}
& z=s \mathcal{E} \\
& \lambda=\left(\frac{s}{r}\right)^{1 / \ln (\varepsilon s / r)}, \quad(\lambda \in(1, e)) .
\end{aligned}
$$

Then $z \in[x / \varepsilon, y \varepsilon]$ and $s=z^{\ln \lambda} r^{1-\ln \lambda}$, and we obtain

$$
f(s) \leq f(z)^{\ln \lambda} f(r)^{1-\ln \lambda}=\left(\frac{f(z)}{f(r)}\right)^{\ln \lambda} f(r)
$$

which yields

$$
\begin{aligned}
\ln \left(\frac{f(s)}{f(r)}\right) & \leq \ln \lambda \ln \left(\frac{f(z)}{f(r)}\right) \\
& <\ln \left(\frac{s}{r}\right)^{1 / \ln (\varepsilon)} \ln \left(\frac{M}{m}\right), \\
\frac{f(s)}{f(r)} & \leq\left(\frac{s}{r}\right)^{\ln (M / m) / \ln (\varepsilon)},
\end{aligned}
$$

where $K=\ln (M / m) / \ln (\varepsilon)>0$. Since the points $r, s \in[x, y]$ are arbitrary, we get $f$ that satisfies a multiplicative Lipschitz condition. The remaining part can be obtained in the similar way by taking $s<r$ and $z=s / \varepsilon$. Finally, $f$ is continuous, since $[x, y]$ is arbitrary in $I^{0}$.

\section{Concluding Remarks}

Although all arithmetics are isomorphic, only by distinguishing among them do we obtain suitable tools for constructing all the non-Newtonian calculi. But the usefulness of arithmetic is not limited to the construction of calculi; we believe there is a more fundamental reason for considering alternative arithmetics; they may also be helpful in developing and understanding new systems of measurement that could yield simpler physical laws.

In this paper, it was shown that, due to the choice of generator function, $A_{p}, G_{p}$, and $H_{p}$ means are reduced to ordinary arithmetic, geometric, and harmonic mean, respectively. As shown in Table 1 , for increasing values of $p, A_{p}$ and $\widetilde{A}_{p}$ means increase, especially $p \rightarrow \infty$; these means converge to the value of $\max \left\{x_{n}\right\}$. Conversely for increasing values of $p, H_{p}$ and $\widetilde{H}_{p}$ means decrease, especially $p \rightarrow \infty$; these means converge to the value of $\min \left\{x_{n}\right\}$. Additionally we give some new definitions regarding convex functions which are plotted on the non-Newtonian coordinate system. Obviously, for different generator functions, one can obtain some new geometrical interpretations of convex functions. Our future works will include the most famous Hermite Hadamard inequality for the class of $*$-convex functions.

\section{Competing Interests}

The authors declare that they have no competing interests.

\section{References}

[1] C. Niculescu and L.-E. Persson, Convex Functions and Their Applications: A Contemporary Approach, Springer, Berlin, Germany, 2006.

[2] C. P. Niculescu, "Convexity according to the geometric mean," Mathematical Inequalities \& Applications, vol. 3, no. 2, pp. 155$167,2000$.

[3] R. Webster, Convexity, Oxford University Press, New York, NY, USA, 1995.

[4] J. Banas and A. Ben Amar, "Measures of noncompactness in locally convex spaces and fixed point theory for the sum of two operators on unbounded convex sets," Commentationes Mathematicae Universitatis Carolinae, vol. 54, no. 1, pp. 21-40, 2013.

[5] J. Matkowski, "Generalized weighted and quasi-arithmetic means," Aequationes Mathematicae, vol. 79, no. 3, pp. 203-212, 2010.

[6] D. Głazowska and J. Matkowski, "An invariance of geometric mean with respect to Lagrangian means," Journal of Mathematical Analysis and Applications, vol. 331, no. 2, pp. 1187-1199, 2007.

[7] J. Matkowski, "Generalized weighted quasi-arithmetic means and the Kolmogorov-Nagumo theorem," Colloquium Mathematicum, vol. 133, no. 1, pp. 35-49, 2013.

[8] N. Merentes and K. Nikodem, "Remarks on strongly convex functions," Aequationes Mathematicae, vol. 80, no. 1-2, pp. 193199, 2010.

[9] M. Grossman, Bigeometric Calculus, Archimedes Foundation Box 240, Rockport, Mass, USA, 1983.

[10] M. Grossman and R. Katz, Non-Newtonian Calculus, Newton Institute, 1978. 
[11] M. Grossman, The First Nonlinear System of Differential and Integral Calculus, 1979.

[12] A. E. Bashirov, E. M. Kurpınar, and A. Özyapıcı, "Multiplicative calculus and its applications," Journal of Mathematical Analysis and Applications, vol. 337, no. 1, pp. 36-48, 2008.

[13] D. Aniszewska, "Multiplicative Runge-Kutta methods," Nonlinear Dynamics, vol. 50, no. 1-2, pp. 265-272, 2007.

[14] E. Misırlı and Y. Gürefe, "Multiplicative adams-bashforthmoulton methods," Numerical Algorithms, vol. 57, no. 4, pp. 425-439, 2011.

[15] A. F. Çakmak and F. Başar, "Some new results on sequence spaces with respect to non-Newtonian calculus," Journal of Inequalities and Applications, vol. 2012, article 228, 2012.

[16] A. F. Çakmak and F. Başar, "Certain spaces of functions over the field of non-Newtonian complex numbers," Abstract and Applied Analysis, vol. 2014, Article ID 236124, 12 pages, 2014.

[17] S. Tekin and F. Başar, "Certain sequence spaces over the nonNewtonian complex field," Abstract and Applied Analysis, vol. 2013, Article ID 739319, 11 pages, 2013.

[18] U. Kadak and H. Efe, "Matrix transformations between certain sequence spaces over the non-Newtonian complex field," The Scientific World Journal, vol. 2014, Article ID 705818, 12 pages, 2014.

[19] U. Kadak and M. Ozluk, "Generalized Runge-Kutta method with respect to the non-Newtonian calculus," Abstract and Applied Analysis, vol. 2015, Article ID 594685, 10 pages, 2015.

[20] U. Kadak, "Determination of the Köthe-Toeplitz duals over the non-Newtonian complex field," The Scientific World Journal, vol. 2014, Article ID 438924, 10 pages, 2014.

[21] U. Kadak, "Non-Newtonian fuzzy numbers and related applications," Iranian Journal of Fuzzy Systems, vol. 12, no. 5, pp. 117-137, 2015.

[22] Y. Gurefe, U. Kadak, E. Misirli, and A. Kurdi, "A new look at the classical sequence spaces by using multiplicative calculus," University Politehnica of Bucharest Scientific Bulletin, Series A: Applied Mathematics and Physics, vol. 78, no. 2, pp. 9-20, 2016. 


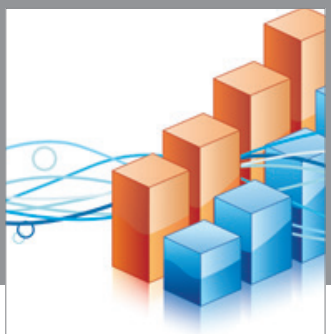

Advances in

Operations Research

vatem alat4

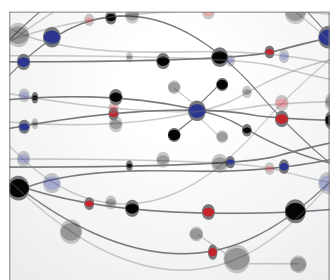

\section{The Scientific} World Journal
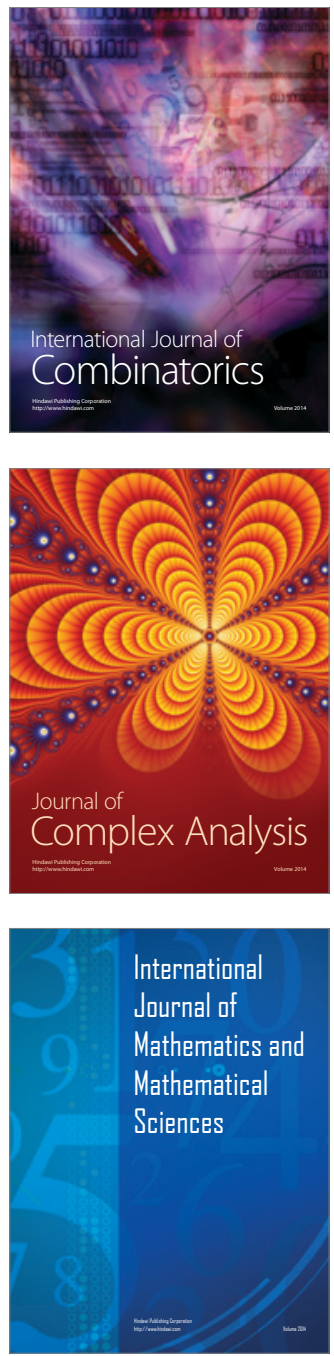
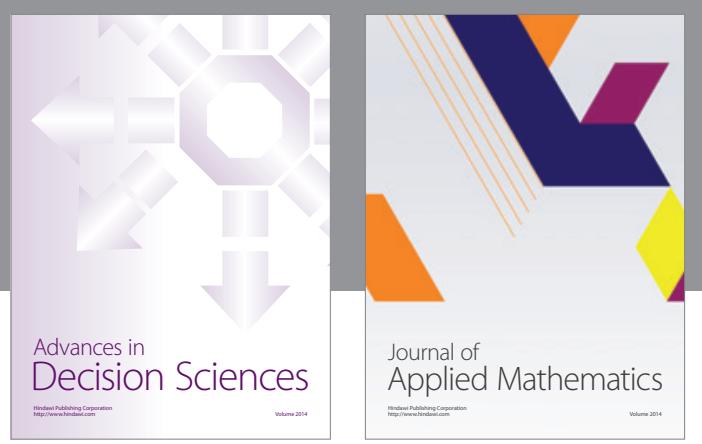

Algebra

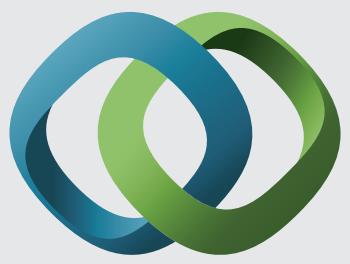

\section{Hindawi}

Submit your manuscripts at

http://www.hindawi.com
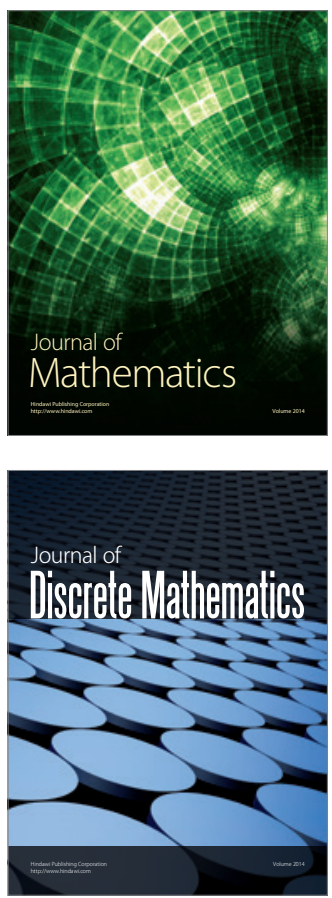

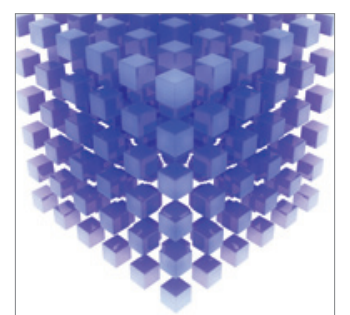

Mathematical Problems in Engineering
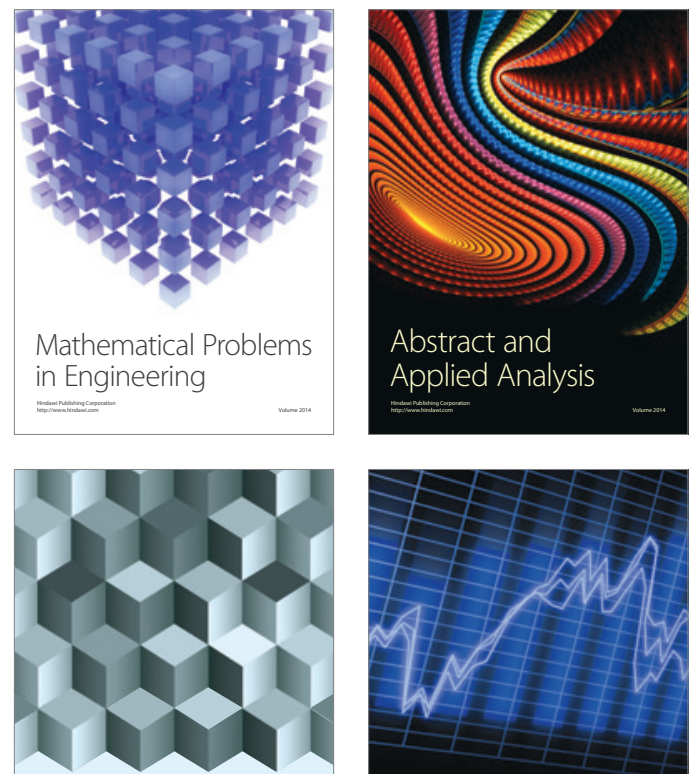

Journal of

Function Spaces

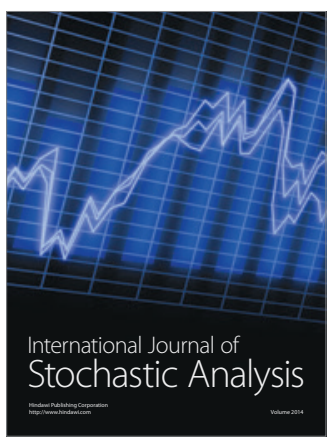

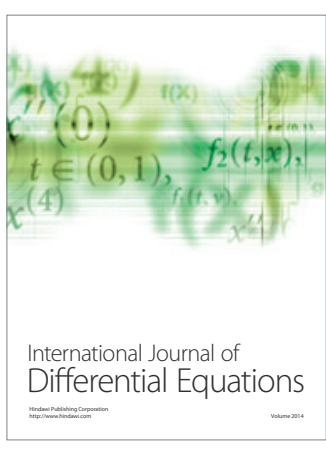
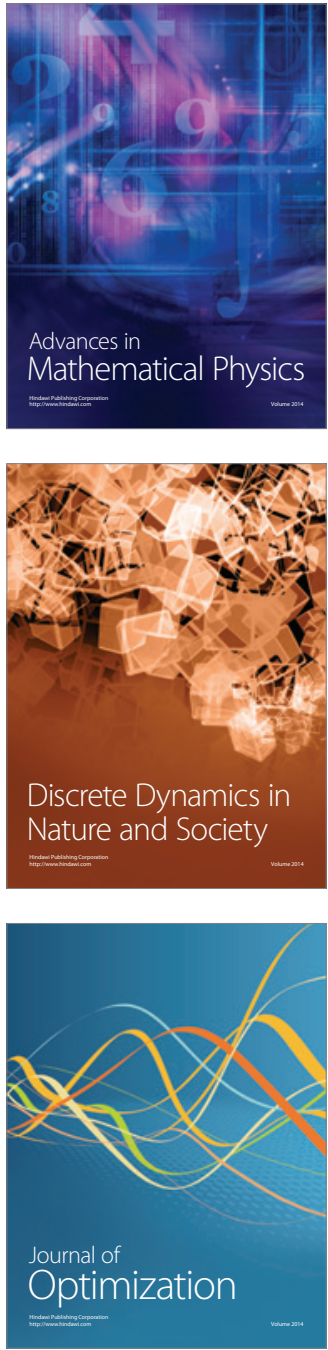